\title{
Determination of Pulse Rate and Blood Pressure Using Non-invasive In-vivo Spectroscopy
}

\author{
Mehedy Hasan, Hoon Kim \\ University of Utah \\ Salt Lake City, Utah, USA \\ mehedy.hasan@utah.edu; hoon.kim@utah.edu
}

\begin{abstract}
Before the first symptoms, high blood pressure (HBP or hypertension) damages human body for years. Statistically half of the untreated hypertension results to fatal heart attack. For years, there have been different types of blood pressure measurement techniques proposed. Despite of not having pin-point accuracy, non-invasive blood pressure (BP) measurement is preferred to invasive measurement techniques due to the facts like avoid bleeding, infections and for simplicity as well. In this paper, a non-invasive in-vivo technique has been described to calculate pulse rate and systolic and diastolic pressure for human body. An image sensor with a novel single carrier modulation photo-detector (SMPD) has been used to capture vein or artery images. Through some video and image processing, pulse rate has been determined with significant accuracy. Some further numerical analysis have been done to calculate systolic and diastolic pressure.
\end{abstract}

Keywords: Pulse Rate, Blood Pressure, Non-invasive, In-vivo, NIR, near-infrared

\section{Introduction \& Literature Review}

The ideal blood pressure for human is $120 / 80$ (systolic/diastolic). With the age these numbers normally go up. High blood pressure is the circumstance when the force of the blood against artery walls is high enough to cause health problems. Normally, the blood pressure is higher when more blood is pumped by heart and arteries are narrow. Hypertension is a long term process. Someone can have it for a long time without any obvious symptoms. Uncontrolled high blood pressure (HBP) increases the risk of health problems like heart attack and stroke. The most important modern day lifestyle factors like cigarette smoking, obesity, too much salt in diet, physical inactivity etc. influence HBP to a great extent.

A number of invasive methods have been available for pulse rate and blood pressure so far. This work has been motivated mostly by some of the recent success for visualization of subsurface blood vessels. The feasibility of infrared video imaging of subsurface stomach vessels was investigated by Gostout et al. [1]. They also revealed subcutaneous venous structure in human arm with infrared light (wavelength>700nm) using infrared sensitive video endoscope. A method of estimating local pulse wave velocity (PWV) was proposed [2] from ultrasound measurement solely as the ratio between pulse pressure and change in cross sectional area. The aortic pressure and common carotid artery diameter waveforms were determined and pressure area curves were described using dual exponential analytical model [3]. Vasculuminator, a device for obtaining access to blood vessels based on transillumination with near infrared light was developed [4]. It is pretty much effective for safe and effective visualisation of subsurface blood vessels to facilitate blood withdrawal in children.

\section{Image Capture}

Blood vessels for peripheral venous and arterial access normally located up to some millimetres below the skin surface. Normally, they are embedded in subcutaneous adipose tissue layer (Depth 1 to several millimetres). The two different kinds of haemoglobin in blood (oxy-haemoglobin and deoxy-haemoglobin) has different absorption spectra. The absorption spectrum shows that, they have peaks at 640nm (deoxy-haemoglobin) and 940nm (oxy-haemoglobin). For water, the better absorption spectra is within the range from 750 to $1500 \mathrm{~nm}$. Molar extinction coefficient for melanin is around $10^{3} \mathrm{~cm}^{-1} \mathrm{M}^{-1}$ near $600 \mathrm{~nm}$. Within the NIR range in spectrum $(700-1400 \mathrm{~nm})$, the absorption by melanin and 
haemoglobin is pretty low. At the same time, the absorption of water over 900nm is high enough for deep tissue penetration. So it can be deduced that, the suitable near infrared window for deep tissue penetration with light is 700 and $1000 \mathrm{~nm}$.

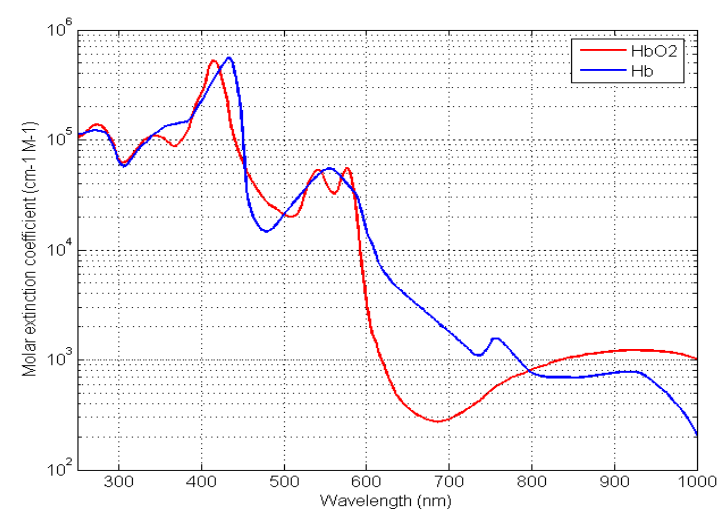

(a)

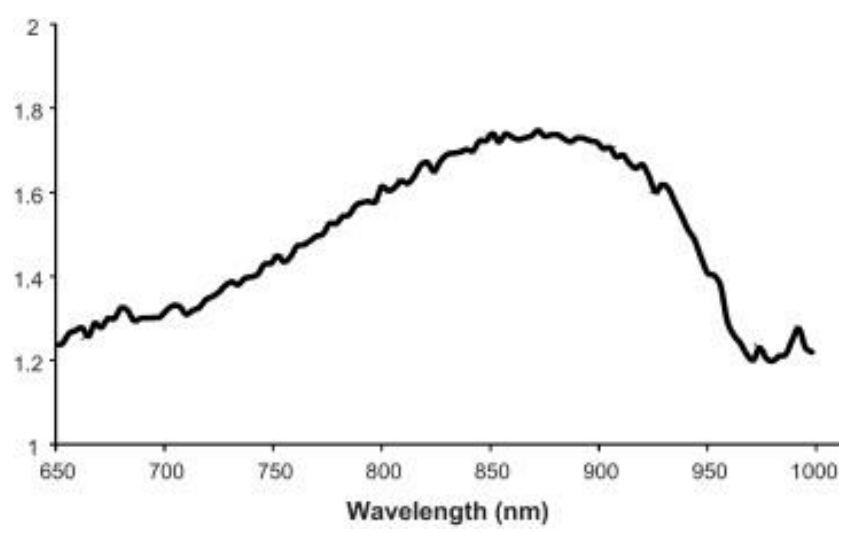

(b)

Fig. 1: (a) Molar extinction coefficient vs. wavelength for oxy-hemoglobin and hemoglobin [5] (b) Contrast index of light intensity at each wavelength between blood vessels and surrounding tissue [4].

\subsection{Sensor and Camera}

A novel structured device was introduced [6] for the application of a unit pixel of CMOS image sensor. It was a single carrier modulation photo detector (SMPD) with p-type MOSFET that has a floating $\mathrm{N}$-well for isolation and wide wavelength absorption. The top layout view and cross sectional structure of the device is shown in figure 2. The SMPD is characterized by wide dynamic range (WDR) and high sensitivity against a conventional photodiode. Proposed SMPD has an energy barrier modulation for the p-channel conductance. That is provided by the amount of photon assisted hotelectron injection into the polycrystalline gate. The vacant site is easily occupied by the localized strong field effect in a line edge of the interface between gate and drain. It can be applied for a unit pixel with high sensitivity and WDR.

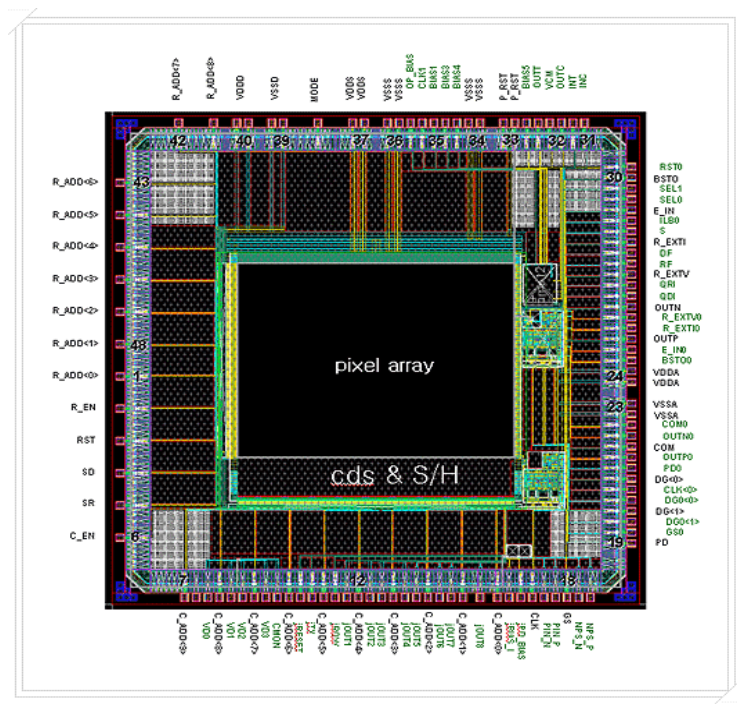

(a)

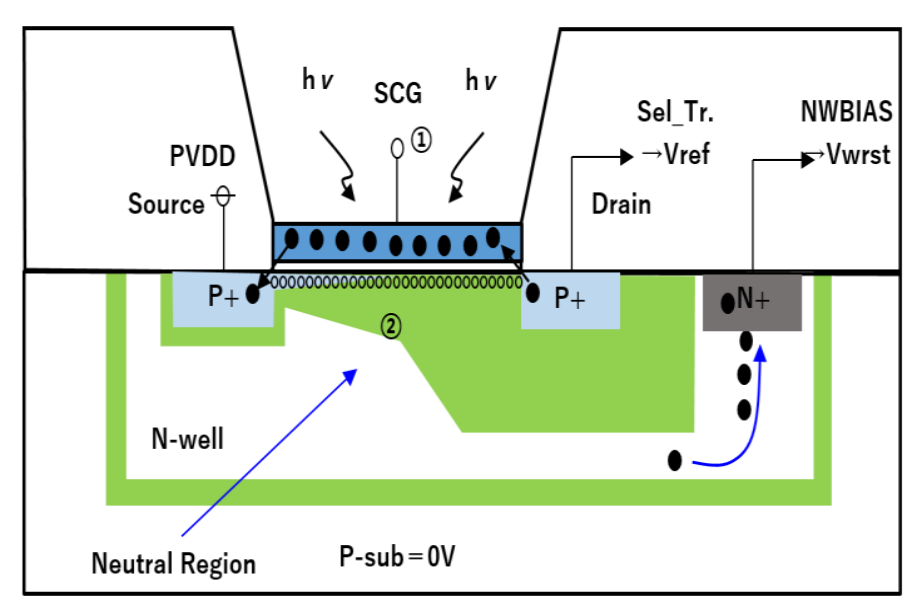

(b)

Fig. 2: Single carrier modulation photo detector (SMPD) (a) Top layout view (b) Cross sectional view. 


\subsection{Capture Setup}

As stated previously, suitable near infrared window for deep tissue penetration with light is between 700 and $1000 \mathrm{~nm}$. The illuminator or light source has been chosen with a wavelength of $850 \mathrm{~nm}$. Two of the most easily accessible blood vessels or arteries are dorsal metacarpal vein (afferent of dorsal venous arch) in wrist and palmer brunches of proper digital arteries in fingers. These were used as image subject. Image capture setup is shown in figure 3. A large illuminator was required for wrist arteries' imaging and single LED was used for palmer brunches in finger. Some of the infrared images are also shown figure 4.

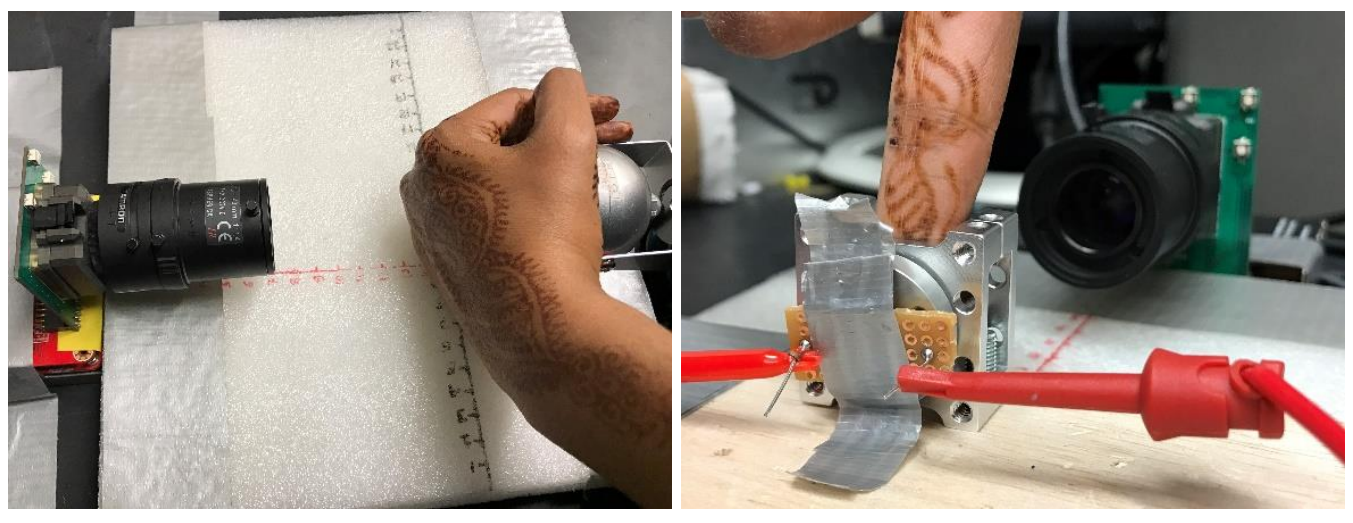

Fig. 3: Capture Setup.

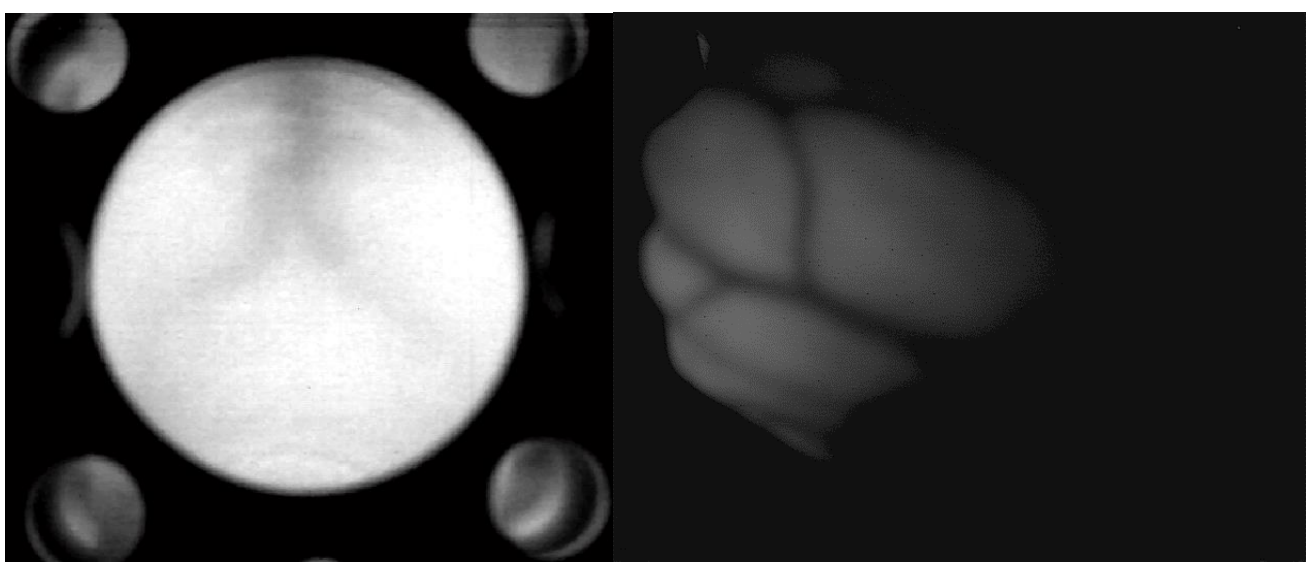

(a)

(b)

Fig. 4: Sample infrared image of blood vessels in (a) finger \& (b) wrist.

\section{Theories \& Mathematics}

\subsection{Pulse Wave Velocity}

There is a very critical role of arterial stiffness in the development of hypertension or any other cardiovascular diseases. Pulse wave velocity is one way to measure the stiffness. It is the rate at which the blood pressure waves move down the vessel. Basically, the softer and the more elastic the aortic wall, the lower the pulse wave velocity (PWV) is. Thus, the lowest pulse wave velocity (PWV) can be measured on the aorta, because this is typical elastic "reservoir" artery. Towards the periphery if the arterial wall contains more muscular elements, the wall will be stiffer, and consequently the pulse wave will be higher and higher.

Pulse wave velocity can be given by the equation [2], 


$$
P W V=\sqrt{\frac{A X P P}{\rho X \triangle A}}
$$

Here, $\mathrm{A}$ is the cross sectional area of the vessel, $\Delta \mathrm{A}$ is the difference between maximum and minimum vein cross sectional areas, PP the pulse pressure, and $\rho$ the blood density.

Table 1: PWV for different age range [7].

\begin{tabular}{|c|c|c|}
\hline Age (years) & Mean PWV (m/s) & $\begin{array}{c}\text { Lower-Upper Limit } \\
\text { (95\% Confidence intervals) }\end{array}$ \\
\hline $10-19$ & 5.04 & $4.92-5.15$ \\
\hline $20-29$ & 5.86 & $5.68-6.03$ \\
\hline $30-39$ & 6.32 & $6.16-6.47$ \\
\hline $40-49$ & 6.85 & $6.68-7.03$ \\
\hline $50-59$ & 8.15 & $7.97-8.33$ \\
\hline $60-69$ & 8.47 & $8.25-8.68$ \\
\hline
\end{tabular}

\subsection{Pressure Calculation}

From equation (1),

$$
P P=(P W V)^{2} \frac{\rho}{A} \Delta A
$$

For a sample measurement (a 26 year old guy) in case of pressure determination, the following information have been extracted from the MATLAB image processing:

Mean cross sectional area $A=\pi(23 r)^{2}$, here, $r=$ proportionality for single pixel magnification (lens magnification).

Difference between maximum and minimum vein cross sectional areas (systolic) $\Delta A=\pi\left[(25 \mathrm{r})^{2}-(20 \mathrm{r})^{2}\right]$

Blood density, $\rho=1025 \mathrm{kgm}^{-3}$

Pulse wave velocity (for 27 year old) $=6.03 \mathrm{~ms}^{-1}$ (upper limit to calculate systolic pressure).

Putting all these in aforementioned equation (2), systolic pressure was approximately $15 \mathrm{Kpa} \sim 112 \mathrm{mmHg}$.

Using the same procedure the diastolic pressure was approximately $12.5 \mathrm{Kpa} \sim 92 \mathrm{mmHg}$.

\subsection{Pulse Rate}

A normal resting heart rate, for adult ranges from 60 to 100 beats a minute. Generally, a lower heart rate at rest implies more efficient heart function and better cardiovascular fitness. For example, a well-trained athlete might have a normal resting heart rate closer to 40 beats a minute. There are many factors that can influence heart rate including: activity level, fitness level, air temperature, body position (for example: standing up or lying down), emotions, body size, medications. Although there's a wide range of normal, an unusually high or low heart rate may indicate an underlying problem.

For determination of the pulse rate, infrared images of blood vessels have been taken for a length of duration using the CMOS image sensor. The boundary region of the vein has been observed closely. In other words, the pulse information has been looked for within the fluctuation of the pixel distributions near the blood vessel edge. The pulse rate has been extracted through the periodicity within that fluctuation using Fourier Transform.

\section{Numerical Analysis}

The frame rate for the image sensor is 25 to 40 frames per second. A video has been taken for a duration of 3 seconds with the maximum frame rate of 40 per seconds. Thus, there are a total of 120 frames to deal with in the video. Then it has been analysed in MATLAB. Every frame corresponds to 580 X 760 matrix of pixels. Instead of taking all of them into account, a slice of pixels has been taken across the blood vessel area for the analysis from each frame. So basically, a total of 120 slices of pixels as shown in figure have been analysed corresponding to time (i.e. 
different frames). For the determination of pressure, the cross section area of blood vessel was the main concern for both systolic and diastolic cases. In case of pulse rate determination, the fluctuation of pixels near the boundary of the vessel was the main focus.

As shown in figure 5, the dark pixels (at centre - blue columns) represents the vessel area and lighter pixels indicates the tissue area near surrounding that. Thus the cross section area was determined by the number of dark pixels (horizontally). Difference between the cross sectional areas during systole and diastole was used to calculate the corresponding pressures as shown in previous pressure calculation section. It can be easily observed, centre of the dark areas are pretty much solid i.e. no or very few fluctuations in pixel values. On the other hand, the boundaries on both sides indicates a lot of fluctuations in pixel values. If a single column near any boundary is chosen, it corresponds to pixel fluctuation due to the pulse wave velocity in blood vessels (figure 6(a)). Some elementary filtering was required to get rid of white noises from ambient and sensor or camera setup. Finally, a simple Fourier analysis was done to point out the pulse rate (figure $6(b)$ ).

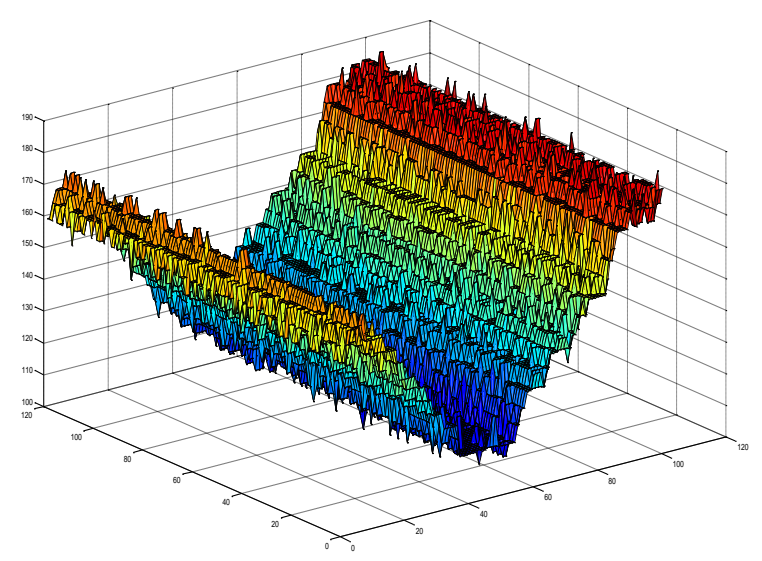

(a)

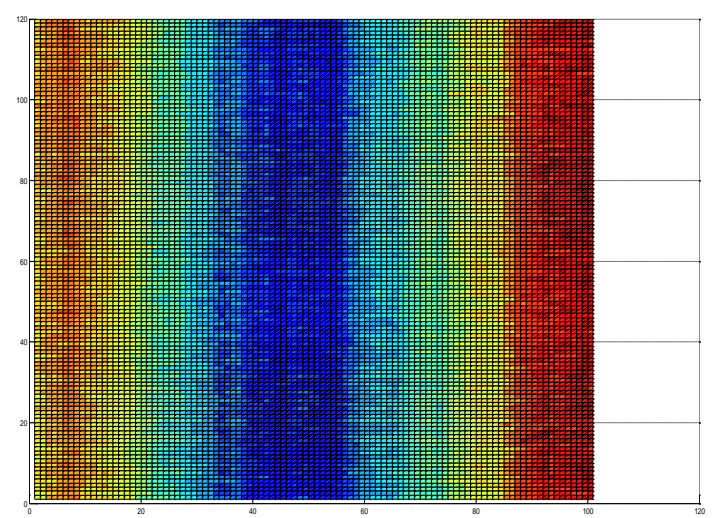

(b)

Fig. 5: Pixel Distributions in 580X760 arrays (a) 3D view (b) top or 2D view. 


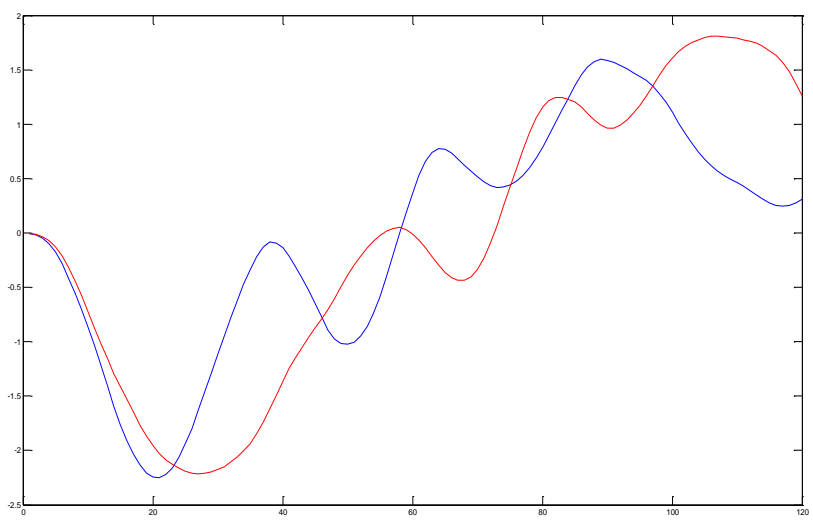

(a)

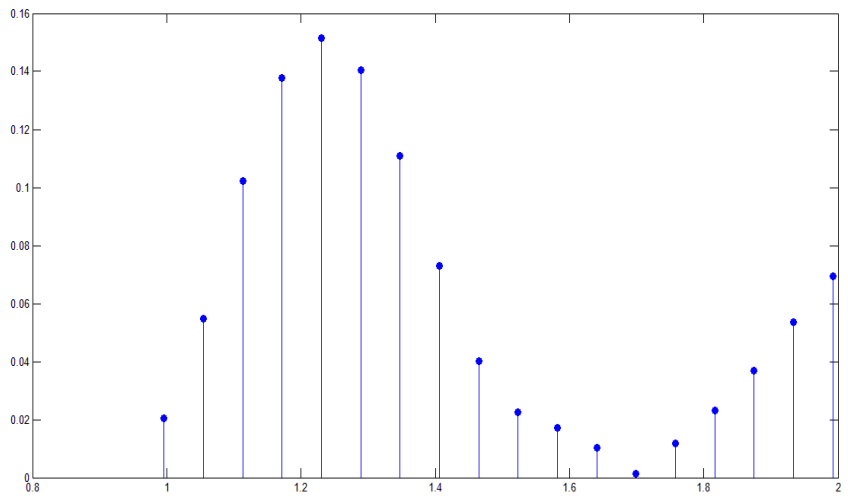

(b)

Fig. 6: Image processing Simulation Results (a) Filtered Fluctuations at Vessel's edge at two different sites and (b) Fourier Transform of it.

\section{Conclusion}

A novel method for non-invasive measurement of blood pressure and pulse rate has been presented. Early detection of high blood pressure is extremely necessary and can save a lot of lives from the threat of blood pressure related diseases. The method explained here is pretty much straight forward and can be interfaced very easily for smartphones. As it is an in-vivo technique, noise involved with CMOS sensors, light source and from ambient as well should be taken into account for accurate determination of pulse rate and blood pressure. The effect of noise can easily be enervated with appropriate filtering during the image or video processing.

\section{Acknowledgements}

We would like to thank Professor Carlos Mastrangelo (USTAR Professor, ECE Faculty, University of Utah) for our discussions on different sensors as well as video and image processing.

\section{References}

[1] C. J. Gostout, and S. L. Jacques, "Infrared video imaging of subsurface vessels: a feasibility study for the endoscopic management of gastrointestinal bleeding," Gastrointestinal endoscopy, vol. 41, no. 3, pp. 218-224, 1995.

[2] S. I. Rabben, et al, "An ultrasound-based method for determining pulse wave velocity in superficial arteries," Journal of biomechanics, vol. 37, no. 10 pp. 1615-1622, 2004.

[3] E. Hermeling, et al, "Noninvasive assessment of arterial stiffness should discriminate between systolic and diastolic pressure ranges," Hypertension, vol. 55, no. 1, pp. 124-130, 2010. 
[4] N. J. Cuper, et al, "The use of near-infrared light for safe and effective visualization of subsurface blood vessels to facilitate blood withdrawal in children," Medical engineering \& physics, vol. 35, no. 4, pp. 433-440, 2013.

[5] S. Prahl, "Optical absorption of haemoglobin," Oregon Medical Laser Center, 1999, [Online]. Available: http://omlc.ogi.edu/spectra/hemoglobin/index.html

[6] H. Kim, S. I. Chang, M. K. Je, H. C. Shin, and Y. Nagamune: Ext. Abst, Int. Conf. Solid State Devices and Materials, Business Center for Academic Societies, Japan, Tokyo, p. 294, 2001.

[7] A. Díaz, et al. "Reference values of pulse wave velocity in healthy people from an urban and rural Argentinean population," International journal of hypertension, 2014. 